\title{
ON THE SEMIPRIMITIVITY OF FINITELY GENERATED ALGEBRAS
}

\author{
JAN OKNIŃSKI
}

(Communicated by Birge Huisgen-Zimmermann)

\begin{abstract}
Finitely generated associative algebras $A=K\left\langle a_{1}, \ldots, a_{n}\right\rangle$ over a field $K$ defined by homogeneous relations are considered. If there exists an order on the associated free monoid $\mathrm{FM}_{n}$ of rank $n$ such that the set of normal forms of elements of $A$ is a regular language in $\mathrm{FM}_{n}$, then the algebra $A$ is semiprimitive provided that the associated monomial algebra is semiprime.
\end{abstract}

Automaton algebras were defined by Ufnarovskii by the condition that the set of normal forms of elements of the algebra is a regular language; see [16]. More precisely, if $A$ is a finitely generated algebra over a field $K$ with a set of generators $a_{1}, \ldots, a_{n}$, then let $K\left\langle x_{1}, \ldots, x_{n}\right\rangle$ denote the free $K$-algebra of rank $n$ and let $\pi: K\left\langle x_{1}, \ldots, x_{n}\right\rangle \longrightarrow A$ be the homomorphism such that $\pi\left(x_{i}\right)=a_{i}$, for $i=$ $1, \ldots, n$. Assume that a well order $\prec$ is given on the free monoid $\mathrm{FM}_{n}=\left\langle x_{1}, \ldots, x_{n}\right\rangle$ such that the unity of $\mathrm{FM}_{n}$ is the least element and that is compatible with the multiplication in $\mathrm{FM}_{n}$. The latter means that $v \prec u$ implies that $v w \prec u w$ and $w v \prec w u$ for all $u, v, w \in \mathrm{FM}_{n}$. Let $I=I_{\pi}$ be the ideal of $\mathrm{FM}_{n}$ consisting of all leading monomials of elements of $\operatorname{ker}(\pi)$. Then the set $N(A)=\mathrm{FM}_{n} \backslash I$ is called the set of normal words corresponding to the chosen presentation of the algebra $A$ and the chosen order on $\mathrm{FM}_{n}$. The minimal set of generators of $I$ is called the set of obstructions. The image $K\left[\mathrm{FM}_{n}\right] / K[I]$ of the free algebra $K\left[\mathrm{FM}_{n}\right]$ is referred to as the monomial algebra associated to $A$ and is an important tool in the combinatorial aspects of finitely generated algebras; see [6, 16. One says that $A$ is an automaton algebra if $N(A)$ is a regular language; see [16, Section 5.10]. Recall that this means that this set is obtained from a finite subset of $\mathrm{FM}_{n}$ by applying a finite sequence of operations of union, multiplication and operation $*$ defined by $T^{*}=\bigcup_{i \geq 1} T^{i}$, for $T \subseteq \mathrm{FM}_{n}$. It is well known that $N(A)$ is a regular language if and only if the set of obstructions is a regular language [16, Theorem 5.2.2]. Therefore, the class of automaton algebras contains the class of algebras with a finite set of obstructions. In other words, if $K\left[\mathrm{FM}_{n}\right] / K[I]$ is a finitely presented algebra, then $A$ is an automaton algebra. Consequently, due to the definition of Gröbner bases, if an algebra $A$ admits a finite Gröbner basis, then $A$ is an automaton algebra; see [16, §2]. For basic results on regular languages and automata theory we refer the reader to $[8$.

Received by the editors April 11, 2012 and, in revised form, February 1, 2013.

2010 Mathematics Subject Classification. Primary 16S15, 16N20; Secondary 16S36, 20M25, $68 \mathrm{Q} 70$.

This work was supported by MNiSW research grant N201 420539. 
There are many results indicating that the class of automaton algebras not only has better computational properties but also several structural properties that behave better than in the class of arbitrary finitely generated algebras. For example, in this context one can refer to results on the Gelfand-Kirillov dimension (see [16. Section 5.10]), results on the radical in the case of monomial automaton algebras ([16. Section 7.6]), results on prime algebras of this type (1]), and also structural results concerned with the special case of finitely presented monomial algebras ([12, Chapter 24] and [13]).

In general, the notion of an automaton algebra depends on the given presentation and it also depends on the chosen order on the corresponding free monoid $\mathrm{FM}_{n}$. These and some other aspects of Ufnarovskii's approach were studied in [10, 11. In particular, a finite presentation of an algebra $A$ does not imply in general that $A$ is an automaton algebra; see for example [10] or [12, Example 24.21]. On the other hand, there are interesting classes of finitely presented algebras that satisfy this property with respect to every degree-lexicographic order on the corresponding $\mathrm{FM}_{n}[2]$.

If $L$ is an ideal in the monoid $\mathrm{FM}_{n}$, then $\mathrm{FM}_{n} / L$ denotes the Rees factor semigroup defined by $\mathrm{FM}_{n} / L=\left(\mathrm{FM}_{n} \backslash L\right) \cup\{0\}$ with the operation $a \circ b=a b$ if $a b \notin L$ and $a b=0$ if $a b \in L$. Clearly, $\mathrm{FM}_{n} / L$ can be identified with the image of $\mathrm{FM}_{n}$ in $K\left[\mathrm{FM}_{n}\right] / K[L]$. By a degree function on the free monoid $\mathrm{FM}_{n}$ we mean a homomorphism $d$ into the additive semigroup of nonnegative integers $\mathbb{N}$ such that $d^{-1}(0)=\{1\}$. This imposes an $\mathbb{N}$-gradation on the free algebra $K\left[\mathrm{FM}_{n}\right]$ with the degree zero component equal to $K$. We say that a finitely generated algebra $A$ is defined by a homogeneous presentation if the kernel of the corresponding homomorphism $\pi: K\left[\mathrm{FM}_{n}\right] \longrightarrow A$ is homogeneous with respect to the gradation on the free algebra $K\left[\mathrm{FM}_{n}\right]$ determined by a degree function on $\mathrm{FM}_{n}$. Clearly, in this case, there is an induced gradation on $A$. By $\mathrm{J}(A)$ we denote the Jacobson radical of $A$. The aim of this note is to prove the following result.

Theorem 0.1. Assume that $A=K\left\langle a_{1}, \ldots, a_{n}\right\rangle$ is an algebra defined by a homogeneous presentation and such that (with respect to some fixed order on the corresponding free monoid $\mathrm{FM}_{n}$ ) the associated monomial algebra $K\left[\mathrm{FM}_{n}\right] / K[I]$ is an automaton algebra. If $K\left[\mathrm{FM}_{n}\right] / K[I]$ is semiprime, then $A$ is semiprimitive.

We note that Theorem 0.1 extends [9, Theorem 3.2] (which deals with the special case where the ideal $I$ comes from a finite Gröbner basis of $A$, whence it is a finitely generated ideal of $\mathrm{FM}_{n}$ ), at the same time providing a simpler proof that does not depend on a description of the radical of a monomial algebra.

Recall that the monomial algebra $K\left[\mathrm{FM}_{n}\right] / K[I]$ is semiprime if and only if the semigroup $\mathrm{FM}_{n} / I$ has no nonzero nilpotent ideals ([12, Proposition 24.3]).

The following is the key observation needed in the proof of the above theorem.

Lemma 0.2. Assume that for an ideal $L$ of $\mathrm{FM}_{n}$ the algebra $R=K\left[\mathrm{FM}_{n}\right] / K[L]$ is an automaton algebra. Then the monoid $\mathrm{FM}_{n} / L$ has finitely many right and finitely many left annihilator ideals. Consequently, every nil subsemigroup of $\mathrm{FM}_{n} / L$ is nilpotent.

Proof. Recall that the set of normal words $N(R)$ is a regular language if and only if its complement $L=\mathrm{FM}_{n} \backslash N(R)$ is a regular language.

Let $w \in \mathrm{FM}_{n} \backslash L$. Suppose that $w$ is a zero divisor modulo $L$ and write $r(w)=$ $\left\{y \in \mathrm{FM}_{n} \mid w y \in L\right\}$. So, $r(w)$ corresponds to the right annihilator of $w$ in $\mathrm{FM}_{n} / L$. 
It is well known that $L$ is a regular language if and only if on $\mathrm{FM}_{n}$ there are finitely many classes of the right syntactic congruence $\rho_{r}$ determined by $L$; see [8, Remark 1.9.1 in Chapter 6]. The latter is defined by

$(u, v) \in \rho_{r}$ if and only if for every $w \in \mathrm{FM}_{n}$ we have: $u w \in L \Longleftrightarrow v w \in L$.

In other words, there are finitely many right ideals of the form $r(w), w \in \mathrm{FM}_{n}$. Therefore, there exist finitely many right annihilator ideals in the monoid $\mathrm{FM}_{n} / L$. A symmetric argument shows that there are finitely many left annihilator ideals in $\mathrm{FM}_{n} / L$.

It is known that if $S$ is a semigroup with a zero element and $S$ satisfies the ascending chain condition on right and on left annihilator ideals, then every nil subsemigroup of $S$ must be nilpotent; see [4, 17.23]. Therefore, the last assertion of the lemma follows.

Proof of Theorem 0.1. Suppose that $A$ is not semiprimitive. By the hypothesis, a gradation by the semigroup of nonnegative integers on the free algebra $K\left[\mathrm{FM}_{n}\right]$ is given and we have a homomorphism $\pi: K\left[\mathrm{FM}_{n}\right] \longrightarrow A$ such that $A$ can be considered with the induced gradation. By a result of Bergman, the radical of a $\mathbb{Z}$-graded algebra is homogeneous (see [5, Theorem 30.28]). Hence, there exists a nonzero homogeneous element $\alpha \in \mathrm{J}(A)$. Moreover, every such element must be nilpotent because it has degree $>0$. Choose an element $\beta$ in $\pi^{-1}(\alpha)$ with the minimal possible leading term with respect to the given order on $\mathrm{FM}_{n}$. Then $\beta=\sum_{i=1}^{m} \lambda_{i} v_{i}$ with $m \geq 1,0 \neq \lambda_{i} \in K$, for some $v_{1}, \ldots, v_{m} \in \mathrm{FM}_{n}$, and we may assume that $v_{1}$ is the leading term of $\beta$. By the definition of $I$ it is then clear that $v_{1} \notin I$. Then, for every $w \in \mathrm{FM}_{n}$ the element $\alpha \pi(w)$ is again a homogeneous element in the radical of $A$, whence it is nilpotent. Since $v_{1} w$ is the leading term of $\beta w=\sum_{i=1}^{m} \lambda_{i} v_{i} w$, it follows easily that $v_{1} w$ is nilpotent modulo $I$. Thus, $v_{1} \mathrm{FM}_{n}$ is a right ideal of $\mathrm{FM}_{n}$ that is nil modulo $I$. From Lemma 0.2 we know that every nil subsemigroup of $\mathrm{FM}_{n} / I$ is nilpotent. Since $K\left[\mathrm{FM}_{n}\right] / K[I]$ is semiprime, it follows that $v_{1} \in I$. This is a contradiction. The result follows.

We conclude with a few natural problems.

Problem 1. Can one skip the hypothesis that the defining relations are homogeneous?

The answer is affirmative if the base field $K$ is nondenumerable. Indeed, in this case $\mathrm{J}(A)$ is a nil ideal by a result of Amitsur (see [14, Lemma 7.1.2]), whence the above proof also works. However, in general, this seems to be an open problem even in cases where the associated monomial algebra is finitely presented.

Problem 2. Assume that $A$ is an arbitrary finitely presented algebra defined by homogeneous relations. Is $\mathrm{J}(A)$ (locally) nilpotent? Is this true at least for every semigroup algebra $A$ ?

It is not known whether the Jacobson radical of a finitely presented algebra always is a nil ideal; see [15] and [3, Problem 1.92]. This is a problem attributed to Amitsur and Latyshev. Moreover, there are no examples of finitely presented algebras whose Jacobson radical is not locally nilpotent. The special case where the algebra is nil and one asks whether it must be nilpotent also is a well known open problem $([17])$. Notice, however, that there exist finitely presented algebras whose radical is not nilpotent ([] $)$. 


\section{REFERENCES}

[1] Jason P. Bell and Pinar Colak, Primitivity of finitely presented monomial algebras, J. Pure Appl. Algebra 213 (2009), no. 7, 1299-1305, DOI 10.1016/j.jpaa.2008.11.039. MR2497577 (2010i:16035)

[2] Ferran Cedó and Jan Okniński, Gröbner bases for quadratic algebras of skew type, Proc. Edinb. Math. Soc. (2) 55 (2012), no. 2, 387-401, DOI 10.1017/S0013091511000447. MR2928501

[3] Dniester notebook: unsolved problems in the theory of rings and modules, Non-associative algebra and its applications, Lect. Notes Pure Appl. Math., vol. 246, Chapman \& Hall/CRC, Boca Raton, FL, 2006, pp. 461-516, DOI 10.1201/9781420003451.axb. Translated from the 1993 Russian edition [MR1310114] by Murray R. Bremner and Mikhail V. Kochetov and edited by V. T. Filippov, V. K. Kharchenko and I. P. Shestakov. MR2203726

[4] Carl Faith, Algebra. II, Ring theory; Grundlehren der Mathematischen Wissenschaften, No. 191, Springer-Verlag, Berlin, 1976. MR0427349 (55 \#383)

[5] G. Karpilovsky, The Jacobson radical of classical rings, Pitman Monographs and Surveys in Pure and Applied Mathematics, vol. 53, Longman Scientific \& Technical, Harlow, 1991. MR.1124405 (93a:16001)

[6] Günter R. Krause and Thomas H. Lenagan, Growth of algebras and Gelfand-Kirillov dimension, revised edition, Graduate Studies in Mathematics, vol. 22, American Mathematical Society, Providence, RI, 2000. MR1721834 (2000j:16035)

[7] Jan Krempa and Jan Okniński, Some examples of affine monoid algebras, Comm. Algebra 40 (2012), no. 1, 78-103, DOI 10.1080/00927872.2010.524685. MR2876291(2012k:16073)

[8] Gérard Lallement, Semigroups and combinatorial applications, Pure and Applied Mathematics; A Wiley-Interscience Publication, John Wiley \& Sons, New York-Chichester-Brisbane, 1979. MR530552 (81j:20082)

[9] H. Li, Recognizing the semiprimitivity of $N$-graded algebras via Gröbner bases, preprint arXiv:1110.2248v1, to appear in Algebra Colloquium.

[10] Jonas Månsson and Patrik Nordbeck, Regular Gröbner bases, J. Symbolic Comput. 33 (2002), no. 2, 163-181, DOI 10.1006/jsco.2001.0500. MR.1879379 (2003d:16030)

[11] Jonas Månsson and Patrik Nordbeck, A generalized Ufnarovski graph, Appl. Algebra Engrg. Comm. Comput. 16 (2005), no. 5, 293-306, DOI 10.1007/s00200-005-0178-8. MR2233759 (2007g:16067)

[12] Jan Okniński, Semigroup algebras, Monographs and Textbooks in Pure and Applied Mathematics, vol. 138, Marcel Dekker Inc., New York, 1991. MR.1083356 (92f:20076)

[13] Jan Okniński, Structure of prime finitely presented monomial algebras, J. Algebra 320 (2008), no. 8, 3199-3205, DOI 10.1016/j.jalgebra.2008.08.003. MR2450723(2009m:16053)

[14] Donald S. Passman, The algebraic structure of group rings, Pure and Applied Mathematics, Wiley-Interscience [John Wiley \& Sons], New York, 1977. MR 470211 (81d:16001)

[15] Agata Smoktunowicz, Some results in noncommutative ring theory, International Congress of Mathematicians. Vol. II, Eur. Math. Soc., Zürich, 2006, pp. 259-269. MR2275597 (2007m:16002)

[16] V. A. Ufnarovskij, Combinatorial and asymptotic methods in algebra [MR1060321 (92h:16024)], Algebra, VI, Encyclopaedia Math. Sci., 57, Springer, Berlin, 1995, pp. 1-196. MR 1360005

[17] Efim Zelmanov, Some open problems in the theory of infinite dimensional algebras, J. Korean Math. Soc. 44 (2007), no. 5, 1185-1195, DOI 10.4134/JKMS.2007.44.5.1185. MR2348741 (2008g:16053)

Institute of Mathematics, Warsaw University, Banacha 2, 02-097 Warsaw, Poland

E-mail address: okninski@mimuw.edu.pl 